\title{
Testing for synchrotron self-absorption in GRB 970111
}

\author{
A. Crider and E.P. Liang \\ Department of Space Physics and Astronomy, 6100 S. Main, Rice University, Houston, TX 77005-1892, U.S.A.
}

Received December 18, 1998; accepted January 28, 1999

\begin{abstract}
The time-resolved spectra of several gammaray bursts have shown that near the beginning of the burst, some absorption mechanism makes the spectra inconsistent with the simple optically-thin synchrotron shock model. We fit GRB 970111, which shows strong evidence for such absorption, with a synchrotron shock spectra suffering self-absorption. The synchrotron selfabsorption shape does fit the BATSE time-evolving spectra, although it may be inconsistent with BeppoSAX WFC data. If the turnover just below the spectral break is due to synchrotron self-absorption, a magnetic field strength of $410^{7} \mathrm{G}$ is required.
\end{abstract}

Key words: gamma-rays: bursts - gamma-rays: observations - radiation mechanisms: non-thermal techniques: spectroscopic

\section{Introduction}

The multiwavelength fading counterparts of gamma-ray bursts (GRBs) have been shown to be in agreement with the relativistic blast wave model (Mészáros \& Rees 1993). More predictive variations of this model, such as the synchrotron shock model (Katz 1994; Tavani 1996) are consistent with a small number of time-integrated GRB spectra (Cohen et al. 1997), but fail to explain several time-resolved GRB spectra. In particular, the asymptotic photon slope $\alpha\left(F_{\mathrm{N}} \propto \nu^{\alpha}\right)$ below the spectral break is predicted by the synchrotron shock model to be between $-\frac{2}{3}$ and $-\frac{3}{2}$. This was shown to be inconsistent with timeresolved GRB spectra fit with the Band et al. (1993) GRB function (Crider et al. 1997; Crider et al. 1998). Fitting both the Band GRB function and a broken power-law to over 100 bursts, Preece et al. (1998) found roughly a fourth of time-integrated spectra to be inconsistent with the synchrotron shock predictions, as well.

Send offprint requests to: A. Crider

(acrider@spacsun.rice.edu)

(liang@spacun.rice.edu)
The observed high values of $\alpha$ do not easily differentiate between the many possible absorption mechanisms. However, the evolution of $\alpha$, when fitting spectra with the Band GRB function, may favor saturated Comptonization as the absorption mechanism (Crider et al. 1997). This may well be a result of extracting the photon spectra assuming a Band GRB function. In this paper, we fit the time-resolved BATSE LAD spectra of GRB 970111 directly with a self-absorbed synchrotron shock function. We choose this burst because it was very bright, it was seen by many instruments including BeppoSAX and BATSE (trigger 5773), and it had a very high $\alpha=+1.5 \pm 0.2$ (Crider et al. 1998).

\section{Procedures}

We began our analysis of this burst by examining HER/HERB data from BATSE (Fishman et al. 1989) LAD 0, which is publically available from the Compton Observatory Science Support Center. This detector is the most normal to the burst $\left(\theta=11.576^{\circ}\right)$. The burst is also $132^{\circ}$ away from the geocenter, which helps reduce complications from Earth scatter. Our HER/HERB data is available spanning an energy range of $24 \mathrm{keV}$ to $1996 \mathrm{keV}$ and time interval of 0.03 to $21.8 \mathrm{~s}$. The burst lasted for just over $40 \mathrm{~s}$ in this energy range, so that the two last pulses are excluded from our analysis.

At its finest resolution, with bins approximately covering $0.3 \mathrm{~s}$, the signal-to-noise ratio is sufficiently high $(S / N>55)$ that it was possible to fit time-resolved spectra. We did this using the WINGSPAN analysis software. Examining the time-evolving spectra of GRB 970111 (see Fig. 1 of Crider et al. 1998), we have found that during the first $\sim 10 \mathrm{~s}$, the asymptotic slope below the spectral break $\alpha$ is positive and inconsistent with the predictions of the unabsorbed synchrotron shock model, namely $-\frac{3}{2} \leq \alpha \leq-\frac{2}{3}$ (Katz 1994). To overcome this inconsistency, we previously included a photoelectric absorption term to account for the steep low-energy spectra (Böttcher et al. 1999). While this produced marginally acceptable fits to the BATSE data, the required ISM H 


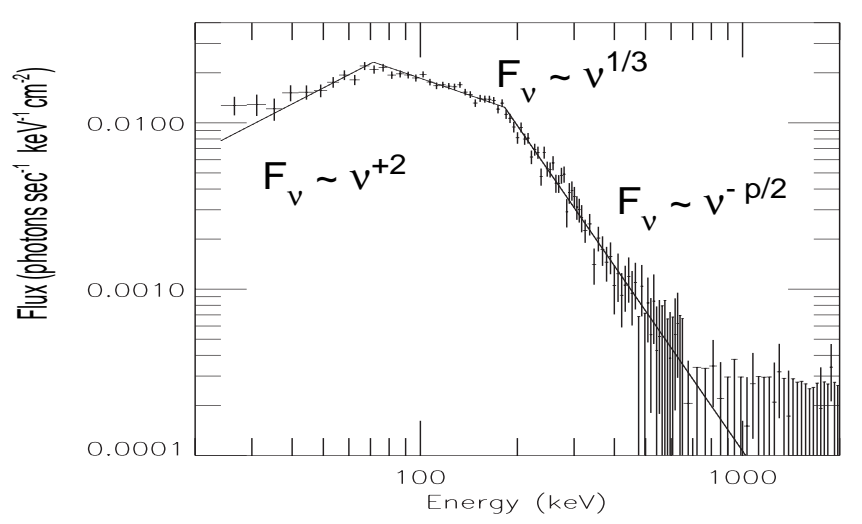

Fig. 1. A simple synchrotron self-absorption model fit to the first $5 \mathrm{~s}$ of GRB $970111\left(\chi_{\nu}^{2}=1.09, \nu=115\right)$

column density is (assuming solar abundances) approximately $10^{26} \mathrm{~cm}^{-2}$ and no photons would have been detected by the BeppoSAX WFC.

Synchrotron self-absorption (SSA) is another possible mechanism which may explain the paucity of photons just below the spectral break. We approximated SSA using a broken power law with two breaks. We fixed the photon slope below the first break to +1 (Katz 1994) and the slope between the two breaks to $-\frac{2}{3}$ (for the slope expected for single electron emission synchrotron shocks; Katz 1994). This leaves 4 free parameters. The resulting $\chi^{2}$ values are similar to those found when using the Band GRB function. In Fig. 1, we show the integrated spectra during the first $5 \mathrm{~s}$ for this burst fit with our simple SSA function. The reduced $\chi^{2}(\nu=115)$ of this fit is 1.09. Fitting this function to the time-resolved spectra reveals that the lower break energy $E_{\text {abs }}$ decreases monotonically while it is within the range of the detector (see Fig. 2).

For fully radiative shock evolution, $E_{\text {abs }} \propto \mathrm{t}^{-\frac{4}{5}}$, while for fully adiabatic shock evolution, $E_{\text {abs }} \propto \mathrm{t}^{-\frac{1}{2}}$ (Sari et al. 1998). To compare the observed decay to these predictions, we fit our data with the function

$E_{\text {abs }}=E_{\text {abs }}(0)\left[1+\left(\frac{t}{t_{\text {decay }}}\right)^{n}\right]^{-1}$

which becomes $E_{\text {abs }} \propto t^{-n}$ when $\left(t / t_{\text {decay }}\right)^{n} \gg 1$. We found that this function fits our values of $E_{\text {abs }}$ very well and find that $t_{\text {decay }}=9.3 \pm 0.5$ and $n=2.2 \pm 0.3$.

\section{Discussion}

If the paucity of photons just below $E_{\mathrm{pk}}$ is due to synchrotron self-absorption, the magnetic energy density must be extremely high. From Rybicki \& Lightman (1979, Eq. (6.53)) we find that

$E_{\mathrm{abs}}=C(p) \tau_{\mathrm{T}}^{\frac{2}{\mathrm{p}+4}} B^{\frac{\mathrm{p}+2}{\mathrm{p}+4}}$

(also see Liang et al. 1997). For convenience, we calculate $C(p)$ for appropriate values of $p$ in Table 1 . Assuming that $\Gamma \approx 1000$ (making the co-moving $E_{\text {abs }} \approx 70 \mathrm{eV}$ ), $\tau_{\mathrm{T}} \approx 1$ and using the Band et al. (1993) GRB function $\beta$ to give

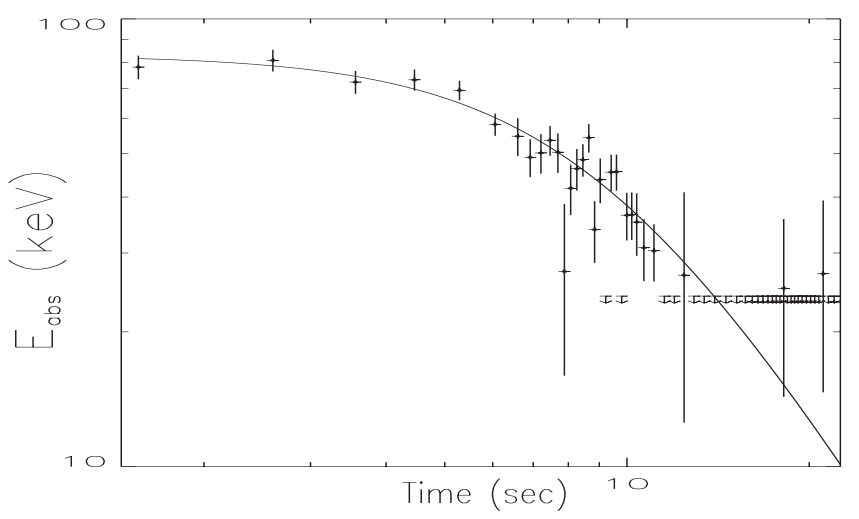

Fig. 2. The decay of $E_{\text {abs }}$ with respect to time fit with Eq. (1). Arrows represent time bins where $E_{\text {abs }}$ was undetermined and presumed to be below the low energy detector cutoff

$p \approx 4$, we find that $B=410^{7} \mathrm{G}$, which constrains some GRB models. Finally, we note that the BeppoSAX WFC data $(2-40 \mathrm{keV})$ for this burst will be very useful in eliminating possible absorption mechanisms once they are released.

Table 1. Calculated values of $C(p)$ for use with Eq. (2). See Rybicki \& Lightman (1979) for details on calculating $C(p)$

\begin{tabular}{ll|ll}
\hline$p$ & $C(p)$ & $p$ & $C(p)$ \\
\hline 2.0 & $1.810^{-3} \mathrm{eV} \mathrm{G}^{-2 / 3}$ & 4.0 & $1.410^{-4} \mathrm{eV} \mathrm{G}^{-3 / 4}$ \\
2.5 & $8.410^{-4} \mathrm{eV} \mathrm{G}^{-9 / 13}$ & 4.5 & $8.910^{-5} \mathrm{eV} \mathrm{G}^{-13 / 17}$ \\
3.0 & $4.310^{-4} \mathrm{eV} \mathrm{G}^{-5 / 7}$ & 5.0 & $5.910^{-5} \mathrm{eV} \mathrm{G}^{-7 / 9}$ \\
3.5 & $2.410^{-4} \mathrm{eV} \mathrm{G}^{-11 / 14}$ & & \\
\hline
\end{tabular}

Acknowledgements. AC thanks NASA-MSFC for his GSRP fellowship. This work is supported by NASA grant NAG5-3824.

\section{References}

Band D., et al., 1993, ApJ 413, 281

Böttcher M., Dermer C., Crider A., Liang E.P., 1999, A\&A 343,111

Cohen E., Katz J.I., Piran T., Sari R., Preece R.D., Band D.L., 1997, ApJ 488, 330

Crider A., Liang E.P., Preece R.D., 1998, AIP 428, 359

Crider A., et al., 1997, ApJ 479, L39

Fishman G.J., et al., 1989, Proc. of the GRO Science Workshop, 2-39

Katz J.I., 1994, ApJ 432, L107

Liang E.P., Smith I.A., Kusunose M., Crider A., 1997, ApJ 479, L35

Preece R.D., et al., 1998, ApJ 506, L23

Mészáros P., Rees M.J., 1993, ApJ 405, 278

Rybicki G.B., Lightman A.P., 1979, Radiative Processes in Astrophysics. New York: Wiley

Sari R., Piran T., Narayan R., 1998, ApJ 497, L17

Tavani M., 1996, ApJ 466, 768 